\title{
THE EFFECTS OF INTERACTIONS ON SPIRAL GALAXIES. II. DISK STAR-FORMATION RATES
}

\author{
ROBERT C. KENNICUTT, JR. ${ }^{\text {a) }}$ \\ Department of Astronomy, University of Minnesota, 116 Church Street, S. E., Minneapolis, Minnesota 55455 \\ William C. KeEL \\ Kitt Peak National Observatory, National Optical Astronomy Observatories, P. O. Box 26732, Tucson, Arizona 85726-6732 \\ and \\ Sterrewacht Leiden, Postbus 9513, 2300 RA Leiden, The Netherlands \\ J. M. VAN DER HULST ${ }^{\text {a) }}$ \\ Netherlands Foundation for Radio Astronomy, Westerbork Radio Observatory, Schattenberg 4, 9433 TA Zwiggelte, The Netherlands \\ E. HuMMEL ${ }^{\text {a) }}$ \\ Max-Planck-Institut für Radioastronomie, Auf dem Hügel 69, D-5300 Bonn 1, Federal Republic of Germany \\ Kurt A. Roettiger \\ Department of Astronomy, University of Minnesota, 116 Church Street, S. E., Minneapolis, Minnesota 55455 \\ Received 23 December 1986
}

\begin{abstract}
We have used $\mathrm{H} \alpha$ emission-line and IRAS far-infrared observations of interacting spiral and irregular galaxies to assess the influence of interactions on their global star-formation rates. Two samples of interacting galaxies were observed: a complete sample of close pairs, and an Arp atlas sample of peculiar systems. When compared to a control sample of single galaxies, both samples of interacting systems exhibit systematically higher levels of both $\mathrm{H} \alpha$ and infrared emission on average, and a larger dispersion in emission properties. A large fraction of the galaxies in the complete-pairs sample exhibit normal starformation properties, but the Arp atlas sample is heavily biased to the most active star-forming systems. Emission levels in the very active systems are much more strongly correlated with the properties of the interaction than with the internal properties of the galaxies themselves. Strong disk emission is almost always accompanied by unusually strong nuclear activity. Simple star-formation burst models can reproduce the observed $\mathrm{H} \alpha$ equivalent widths and broadband colors of most of the galaxies. The bursts are relatively short (few times $10^{7} \mathrm{yr}$ ) and rarely involve more than $1 \%-2 \%$ of a galaxy's total mass. The initial mass function in the strongest bursts is probably systematically enriched in massive stars.
\end{abstract}

\section{INTRODUCTION}

A large fraction of the 'starburst' galaxies revealed by ultraviolet, infrared, and optical emission-line surveys are members of interacting systems (e.g., Heidmann and Kalloghlian 1973; Soifer et al. 1984; Wasilewski 1983; respectively), and this has led several workers to suggest that interactions are an important triggering mechanism for star formation in general. Several comparisons of star-formation activity in isolated and interacting galaxies have yielded conflicting results, however. Strongly interacting galaxies exhibit an unusually large scatter in optical colors (Larson and Tinsley 1978), and often exhibit unusually strong nonthermal radio emission and far-infrared emission (Sulentic 1976; Stocke 1978; Condon et al. 1982; Joseph et al. 1984; Lonsdale et al. 1984; Joseph and Wright 1985), and all of these effects have been attributed to induced star-formation bursts. This interpretation has been questioned, however, by Hummel (1980,1981), who found no systematic difference in the disk radio-emission properties of isolated and interacting galaxies when complete samples were observed.

Much of the ambiguity in these results can be traced to limitations in the observations. The poor spatial resolution of most available data, for instance, has made it difficult to

a) Visiting Astronomer, Kitt Peak National Observatory, National Optical Astronomy Observatories, which are operated by the Association of Universities for Research in Astronomy, Inc., under contract with the National Science Foundation. separate the effects of disk star formation from induced nuclear activity. Nuclear emission is strongly enhanced in interacting galaxies (e.g., Carlson 1977; Hummel 1981; Condon et al. 1982; Joseph et al. 1984; Kennicutt and Keel 1984; Dahari 1984, 1985; Keel et al. 1985; Cutri and McAlary 1985; Bushouse 1986), and spatially resolved observations are required in order to detect any additional enhancement in the disk star-formation rate (SFR). Sample selection effects are also of importance. Many of the observations have been restricted to the most peculiar interacting and merging systems, or to systems which are unusually bright in the radio or infrared. It is not clear whether the star-formation properties of these systems are representative of interacting galaxies in general.

Here we report the results of an optical and infared survey of star formation in interacting spiral and irregular galaxies. High-resolution $\mathrm{H} \alpha$ emission-line maps have been obtained for two sets of galaxies; a complete sample of galaxies with close companions, and a sample of strongly interacting and morphologically peculiar systems. We have combined these data with an earlier survey of $\mathrm{H} \alpha$ emission in normal galaxies (Kennicutt and Kent 1983), and with our published nuclear spectrophotometry (Keel et al. 1985, hereafter referred to as Paper I), in order to objectively evaluate the effects of interactions on the massive star-formation rate (SFR). We have also used the Infrared Astronomical Satellite $(I R A S)$ data to compare the far-infrared (FIR) emission properties of the same galaxies. This paper concentrates on the integrated disk star-formation properties of the galax- 
ies. Further papers in the series will deal with the distribution and luminosities of the star-forming regions, and with the radio-continuum properties of the sample.

\section{DATA}

\section{a) Sample Selection}

Following Paper I, we have defined two samples of interacting galaxies; a complete sample of galaxies with close companions, defined independently of any morphological peculiarities, and an intentionally biased sample of unusually disturbed systems.

Our 'complete' sample was drawn from a catalog of galaxy associations compiled by T. S. van Albada (1986). He used positions and magnitudes from the UGC catalog (Nilson 1973 ) to isolate apparent pairs and groups, and to compute the statistical probability that the associations are real. We selected a magnitude-limited subsample of 50 galaxies which met the following criteria: Hubble type later than S0, association probability $\geqslant 90 \%$, companion redshift within $\Delta v \leqslant 600$ $\mathrm{km} \mathrm{s}^{-1}, 8^{\mathrm{h}}<\alpha<16^{\mathrm{h}}, \delta>0^{\circ}$, and $B_{T}<13.0$ (de Vaucouleurs et al. 1976). The strict selection criteria ensure that chance optical superpositions are negligible. This sample is virtually identical to the "complete sample" in Paper I, and details of its properties may be found there.

Our second sample was drawn from the Arp (1966) Atlas of Peculiar Galaxies. It includes the 29 members of the complete sample that are also in the Arp atlas, and 32 other galaxies selected on the basis of visible tidal distortions, peculiar morphology, and/or evidence from Paper I for unusual nuclear properties. These members of this 'Arp' sample are very similar to the interacting galaxies studied by Larson and Tinsley (1978), Joseph et al. (1984), Lonsdale et al. (1984), and Bushouse (1986).

Our two samples should provide a guide to the star-formation properties of galaxies in complementary regimes. The complete sample provides an objective assessment of the effects of the presence of a close (typical projected separations 0-5 radii) companion on the star formation in a galaxy, averaged over the duration of the encounter. We have deliberately chosen not to impose any further morphological criteria for 'interaction' on these systems, such as tidal distortion or unusual disk structure or surface brightness. The former are only strong for certain orbital geometries, and the latter are strongly dependent on the very enhancements in star formation that we hope to quantify. The Arp sample, in contrast, is strongly biased to just these types of peculiar systems. The properties of this sample should provide an indication of how the star formation may be enhanced under more extreme conditions. It may indicate what happens during unusually strong interactions which are too rare to be represented much in the complete sample, but it will also be biased to unusually active systems, and to the most active stages of more ordinary encounters.
Our primary comparison sample is the $\mathrm{H} \alpha$ survey of Kennicutt and Kent (1983), with E-S0 galaxies and members of close pairs excluded. Although it is not a complete magnitude-limited sample, this 'KK' sample of 140 galaxies covers a representative range of morphological types and luminosities (see Table I). For additional comparison we also obtained FIR data for the magnitude-limited sample of Keel (1983), again with members of close pairs excluded. As will be discussed later, the star-formation properties of the two control samples are very similar.

Table I summarizes the Hubble type distributions, average absolute magnitudes, and average redshifts in the four samples. It is possible that the morphological types of the most perturbed systems have been altered by the interactions themselves, and the substantial number of I0 galaxies in the pair samples may be evidence for this effect. In order to minimize this problem, we generally excluded from the Arp sample extremely disrupted galaxies, such as ring galaxies or mergers. Overall, the complete-pairs sample appears to be well matched to the KK control sample, so we should be able to interpret its star-formation properties directly. The members of the Arp sample possess a similar distribution of morphological types, but are more distant and luminous on average. As a result, we shall base most of our analysis of their properties on luminosity-independent indices of the star-formation activity.

\section{b) Optical Data}

We chose as our primary star-formation indicator the total $\mathrm{H} \alpha$ emission of a galaxy. This has proven to be a sensitive direct tracer of the massive SFR (Kennicutt 1983; Gallagher et al. 1984), and data are available for a large number of normal galaxies from the surveys of Cohen (1976), Kennicutt and Kent (1983), and Kennicutt et al. (1984).

Most of the $\mathrm{H} \alpha$ data for the interacting galaxies were obtained on the $2.1 \mathrm{~m}$ telescope at Kitt Peak National Observatory (KPNO), using an ISIT video camera ( 53 galaxies), or a Texas Instruments CCD direct camera ( 27 galaxies). Each of these instruments produces a photon-limited photometric image over a field of 140" (ISIT) to 160" (CCD) square. Each galaxy (or close pair) was imaged through two interference filters, one centered on the redshifted $\mathrm{H} \alpha$ and [N II] emission lines (filter FWHM 60-80 $\AA$ ), and one located in the stellar continuum at $5800-6200$ or $6350-6450 \AA$. Calibration of the images, consisting of bias subtraction, flatfield division, and (for the ISIT data) distortion correction, was performed using the software at KPNO. Finally, continuum-free emission-line images of the galaxies were produced by subtracting the continuum maps from the observed $\mathrm{H} \alpha$ maps, using known continuum sources such as foreground stars or the spheroidal regions of the galaxies for normalization of the continuum. Figure 1 [Plate 63] illustrates the continuum and net $\mathrm{H} \alpha+[\mathrm{N}$ II] CCD images for NGC

TABLE I. Properties of the samples.

\begin{tabular}{|c|c|c|c|c|c|}
\hline & & Complete pairs & Arp pairs & KK control & Keel control \\
\hline Hubble types & $\begin{array}{l}\text { SOa-Sa } \\
\text { Sab-Sb } \\
\text { Sbc-Sd } \\
\text { Sm-Im } \\
\text { I0 }\end{array}$ & $\begin{array}{r}16 \% \\
24 \% \\
40 \% \\
12 \% \\
8 \%\end{array}$ & $\begin{array}{r}15 \% \\
21 \% \\
34 \% \\
20 \% \\
5 \%\end{array}$ & $\begin{array}{l}13 \% \\
21 \% \\
53 \% \\
13 \% \\
-\end{array}$ & $\begin{array}{r}11 \% \\
39 \% \\
48 \% \\
1 \% \\
1 \%\end{array}$ \\
\hline $\begin{array}{l}\text { Median velocity, } \\
\text { Median } M_{B}\end{array}$ & $\mathrm{~km} \mathrm{~s}^{-1}$ & $\begin{aligned} & 1700 \\
- & 20.4\end{aligned}$ & $\begin{array}{c}3100 \\
-21.1\end{array}$ & $\begin{array}{r}1300 \\
-20.7\end{array}$ & $\begin{aligned} & 1200 \\
- & 20.5\end{aligned}$ \\
\hline
\end{tabular}


5350, a member of the complete sample, and for the pair NGC 5754 + Arp 297C. (Examples of the ISIT images are illustrated in Paper I.) The effective noise of the CCD images is approximately 5 times lower than for comparable ISIT exposures, and hence we reobserved several galaxies from Paper I with the CCD camera.

Data for an additional 11 galaxies in six systems were obtained using a GEC CCD camera on the $1 \mathrm{~m}$ Kapteyn telescope of the Observatorio del Roque de los Muchachos, La Palma. The technique was similar to that used for the KPNO observations, except that a broadband $R$ filter was used for the continuum subtraction. Contamination of the $R$ frame by line emission was removed in the data reduction.

The images contain considerable information on the amounts and the distributions of massive star formation of the galaxies, but we confine the analysis in this paper to the integrated emission properties. The integrated $\mathrm{H} \alpha+[\mathrm{N} \mathrm{II}]$ and continuum fluxes of each galaxy were measured using a digital aperture photometry program, and emission-line equivalent widths (EWs) were derived from the ratio of these fluxes, combined with the known bandpasses of the $\mathrm{H} \alpha$ filters. Each galaxy was measured with an aperture that enclosed as much of the disk as possible, and with 5" and 10" apertures centered on the nucleus. The latter were obtained in order to tie the photometry to the nuclear spectrophotometry in Paper I, and to measure the fraction of the integrated emission that originates in the nucleus and the near-nuclear regions. The reductions were performed at the University of Minnesota, using the AIPS software.

Additional photoelectric aperture photometry was obtained for 22 of the galaxies, using the Schmidt Two-Holer photometer on the UM/UCSD $1.5 \mathrm{~m}$ telescope on Mount Lemmon. Aperture diameters of 32" and an $\mathrm{H} \alpha$ and continuum filter system similar to that described by Kennicutt and Kent (1983) were used for these measurements. The data were used to calibrate the flux scale for many of the images, and served as an independent check on the accuracy of the EWs derived from the imagery. We also used the large-aperture photometry of Kennicutt and Kent (1983) for a few large, nearby galaxies in the complete-pairs sample.

The $\mathrm{H} \alpha+[\mathrm{N}$ II $]$ EWs for the interacting galaxies are listed in Table II. Note that the measurements include the emission of $\mathrm{H} \alpha$ and the satellite [N II] $\lambda \lambda 6548,6584$ lines, but in

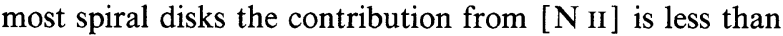
$25 \%$, with a relatively small dispersion among galaxies of the same type (Kennicutt and Kent 1983), so in the discussion that follows we shall often refer to ' $\mathrm{H} \alpha$ ' alone for convenience. The dominant sources of uncertainty in the EWs are errors in the sky background and continuum subtraction. Comparison of EWs derived from the images with the Mount Lemmon aperture photometry indicates that the typical uncertainties are $\pm 3-5 \AA$, or $\pm 10 \%$ for galaxies with very strong emission.

Table II also lists the approximate $\mathrm{H} \alpha+[\mathrm{N}$ II $]$ fluxes. For galaxies observed under photometric conditions, the fluxes were determined directly, using observations of KPNO spectrophotometric standard stars. Otherwise, they were derived by tying the ISIT/CCD instrumental fluxes to the Mount Lemmon photometry, or to the nuclear spectrophotometry in Paper I. In each case simulated aperture photometry was performed on the $\mathrm{H} \alpha$ images, using an aperture that was identical to that used in the photoelectric measurements. As a final check, we also computed a flux from the measured equivalent widths and published blue magnitudes (de Vaucouleurs et al. 1976), using the average relationship for the KK measurements. We estimate that the fluxes for most of the galaxies in Table II are accurate to $\pm 15 \%$, but to be conservative we have rounded all values to the nearest 0.1 dex. Fluxes flagged with colons are uncertain to $\pm 25 \%$. Despite the uncertainties in fluxes of individual galaxies, these data are still useful for examining the general characteristics of the star formation in the interacting galaxies.

\section{c) Infrared Data}

Far-infrared (FIR) fluxes of the galaxies were obtained by combining the 60 and $100 \mu \mathrm{m}$ fluxes from the IRAS Point Source Catalog (1985, PSC), and from the IRAS HCON1 Sky Flux Maps (SFMs). PSC fluxes were used for galaxies which are substantially smaller than the survey resolution, while for larger galaxies the fluxes were determined by integrating the SFMs, using the digital aperture photometry program described earlier. A comparison of fluxes derived using both methods shows good agreement for galaxies with $D(25) \leqslant 4^{\prime}$ (diameters from de Vaucouleurs et al. 1976), and hence we have adopted the PSC fluxes for galaxies smaller than 4', and integrated SFM fluxes for larger galaxies. Roughly $35 \%$ of the galaxies in the complete pairs and control samples were large enough to require SFM integrations. PSC fluxes could be used for virtually all of the Arp pairs, but in this case the emission from individual pair members is usually unresolved, as discussed below.

The dominant sources of uncertainty in the SFM fluxes are the sensitivity of the HCON1 survey (for faint galaxies) and the background correction (for large galaxies). A comparison of SFM fluxes and PSC fluxes for small galaxies shows typical scatter of $15 \%-25 \%$, except for very faint sources near the detection limit. We estimate that the SFM fluxes are accurate to $\pm 20 \%$ or better for sources stronger than $10 \mathrm{Jy}$, with the fractional uncertainty increasing rapidly for fainter sources. Other sources of error, such as field distortion near the edges of the SFM fields, are negligible in comparison for all of the galaxies measured. The fluxes of 18 of the interacting systems were further checked by measuring the IRAS Additional Observations (AOs), which consist of multiple slow scans across preselected fields of interest. Fluxes in all bands in which each galaxy (or pair) was detected were derived from the coadded $\mathrm{AO}$ data, using software at the Leiden IRAS data center. Comparison of the AO and PSC fluxes for 15 systems small enough for reliable application of the PSC algorithm shows scatter of $0.4 \mathrm{Jy}$ at 60 $\mu \mathrm{m}$ and $1.5 \mathrm{Jy}$ at $100 \mu \mathrm{m}$. The published PSC fluxes are known to contain small systematic errors for very weak sources, and a comparison of our SFM integrations with the PSC data shows a weak trend which is consistent with such an error. The AO data show no systematic error, in the PSC fluxes for the small sample in common, however, and as a result we chose not to attempt to apply a correction to the PSC data.

The detection rate of the IRAS survey for all of the samples is excellent. If we exclude a few galaxies which lie in parts of the sky not covered by $I R A S$, the detection rates are $92 \%$ and $94 \%$ for galaxies in the KK and Keel control samples, respectively, and $98 \%$ and $100 \%$ for the complete and Arp samples, respectively. For undetected galaxies we have estimated $1 \sigma$ upper limits, and included these limits in the subsequent analyses. Galaxies not covered in the survey were excluded from the analysis.

The 60 and $100 \mu \mathrm{m}$ fluxes were combined to estimate a 
TABLE II. Data for interacting galaxies.

\begin{tabular}{|c|c|c|c|c|c|c|}
\hline NGC/IC & Arp & Type & $\begin{array}{c}W_{\lambda}(\mathrm{H} \alpha+\mathrm{N} \text { II }) \\
(\AA ̊)\end{array}$ & $\begin{array}{c}\log f(\mathrm{H} \alpha+\mathrm{N} \mathrm{II}) \\
\left(\mathrm{erg} \mathrm{cm}^{-2} \mathrm{~s}^{-1}\right)\end{array}$ & $\begin{array}{c}\log f(\text { FIR }) \\
\left(\mathrm{erg} \mathrm{cm}^{-2} \mathrm{~s}^{-1}\right)\end{array}$ & $\log \frac{L(\mathrm{FIR})}{L(B)}$ \\
\hline \multicolumn{7}{|c|}{ a) Complete sample } \\
\hline $\begin{array}{l}2276 \\
2633 \\
2798 \\
2805 \\
2964\end{array}$ & $\begin{array}{r}25 \\
80 \\
283\end{array}$ & $\begin{array}{l}\text { Sc } \\
\text { SBb } \\
\text { SBa } \\
\text { Scd } \\
\text { Sbc }\end{array}$ & $\begin{array}{l}45 \pm 5 \\
25 \pm 4 \\
63 \pm 7 \\
\pm 1 \pm 5\end{array}$ & $\begin{array}{l}-11.5 \\
-12.1 \\
-11.9 \\
-11.5\end{array}$ & $\begin{array}{c}-9.13 \\
-9.07 \\
-8.95 \mathrm{P} \\
-10.03 \\
-9.17\end{array}$ & $\begin{array}{l}0.62 \\
1.04 \\
1.18 \mathrm{P} \\
-0.03 \\
0.65\end{array}$ \\
\hline $\begin{array}{l}2968 \\
2976 \\
3031 \\
3034 \\
3077\end{array}$ & 337 & $\begin{array}{c}\mathrm{I0} \\
\mathrm{Sc} \\
\mathrm{Sab} \\
\mathrm{I} 0 \\
\mathrm{I} 0\end{array}$ & $\begin{array}{c}2 \pm 2 \\
26 \pm 2 \\
\pm \\
55 \pm 10 \\
27 \pm 4\end{array}$ & $\begin{array}{r}-1 \overline{\overline{3}} \\
-1 \overline{\overline{1}}\end{array}$ & $\begin{array}{r}\text { nd } \\
-8.99 \\
-8.42 \\
-7.26 \\
-9.11\end{array}$ & $\begin{array}{c}<-0.46 \mathrm{P} \\
0.31 \\
-0.35 \\
1.40 \\
0.18\end{array}$ \\
\hline $\begin{array}{l}3166 \\
3169 \\
3185 \\
3190 \\
3227\end{array}$ & $\begin{array}{r}316 \\
316 \\
94\end{array}$ & $\begin{array}{l}\mathrm{S} 0 / \mathrm{a} \\
\mathrm{Sa} \\
\mathrm{SBa} \\
\mathrm{Sa} \\
\mathrm{Sa}\end{array}$ & $\begin{array}{c}0 \pm 3 \\
3 \pm 3 \\
4 \pm 3 \\
0 \pm 3 \\
78 \pm 10\end{array}$ & $\begin{array}{r}\overline{\bar{Z}} \\
-12 . \overline{7} \\
-11 . \overline{9}\end{array}$ & $\begin{array}{c}-9.05 \mathrm{P} \\
\mathrm{P} \\
-10.02 \\
-9.44 \mathrm{P} \\
-9.33 \mathrm{P}\end{array}$ & $\begin{array}{l}0.21 \mathrm{P} \\
\mathbf{P} \\
0.19 \\
0.22 \mathrm{P} \\
0.17 \mathrm{P}\end{array}$ \\
\hline $\begin{array}{l}3395 \\
3396 \\
3430 \\
3690 \\
\text { IC } 694\end{array}$ & $\begin{array}{l}270 \\
270 \\
299 \\
299\end{array}$ & $\begin{array}{l}\text { Scd } \\
\text { IBm } \\
\text { Sc } \\
\text { IBm } \\
\text { SBm }\end{array}$ & $\begin{array}{c}84 \pm 6 \\
154 \pm 15 \\
30 \pm 4 \\
238 \pm 30 \\
190 \pm 30\end{array}$ & $\begin{array}{l}-11.4 \\
-11.4 \\
-11.6 \\
-11.3 \\
-11.5\end{array}$ & $\begin{array}{c}-9.26 \mathrm{P} \\
-8.89 \mathrm{P} \\
-8.27 \mathrm{P} \\
\mathrm{P}\end{array}$ & $\begin{array}{c}0.43 \mathrm{P} \\
\mathbf{P} \\
0.67 \mathrm{P} \\
1.69 \mathrm{P} \\
\mathbf{P}\end{array}$ \\
\hline $\begin{array}{l}3786 \\
3788 \\
\text { IC 749 } \\
\text { IC 750 } \\
3991\end{array}$ & $\begin{array}{l}294 \\
294\end{array}$ & $\begin{array}{l}\text { Sa } \\
\text { Sab } \\
\text { SBc } \\
\text { Sab } \\
\text { Im }\end{array}$ & $\begin{array}{c}82 \pm 15 \\
5 \pm 3 \\
51 \pm 10 \\
25 \pm 10 \\
124 \pm 7\end{array}$ & $\begin{array}{l}-12 . \overline{6} \\
=11.9 \\
-12.2: \\
-11.9\end{array}$ & $\begin{array}{c}\bar{z} \\
\overline{\bar{z}} \\
-9.17 \mathrm{P}\end{array}$ & $\begin{array}{l}\bar{z} \\
\bar{z} \\
0.62 \mathrm{P}\end{array}$ \\
\hline $\begin{array}{l}3994 \\
3995 \\
4394 \\
4438 \\
4485\end{array}$ & $\begin{array}{l}313 \\
313\end{array}$ & $\begin{array}{l}\mathrm{Sc} \\
\mathrm{Sm} \\
\mathrm{SBb} \\
\mathrm{Sb}: \\
\mathrm{IBm}\end{array}$ & $\begin{array}{c}74 \pm 10 \\
96 \pm 7 \\
2 \pm 3 \\
\pm \\
60 \pm 6\end{array}$ & $\begin{array}{r}-11.8 \\
-11.7 \\
\overline{-11.6}\end{array}$ & $\begin{array}{c}\mathbf{P} \\
\mathbf{P} \\
-10.07 \\
-9.49 \mathrm{P} \\
-8.52 \mathrm{P}\end{array}$ & $\begin{array}{c}\mathrm{P} \\
\mathrm{P} \\
-0.30 \\
-0.30 \mathrm{P} \\
0.50 \mathrm{P}\end{array}$ \\
\hline $\begin{array}{l}4490 \\
4567 \\
4568 \\
4631 \\
4647\end{array}$ & $\begin{array}{l}281 \\
116\end{array}$ & $\begin{array}{c}\text { SBd } \\
\text { Scd } \\
\text { Scd } \\
\text { SBd } \\
\text { Sc }\end{array}$ & $\begin{array}{l}80 \pm 8 \\
11 \pm 3 \\
20 \pm 5 \\
39 \pm 3 \\
27 \pm 6\end{array}$ & $\begin{array}{l}-10.5^{\mathrm{a}} \\
=12.1^{\mathrm{a}} \\
=11.7^{\mathrm{a}} \\
-10.7^{\mathrm{a}} \\
-11.8^{\mathrm{a}}\end{array}$ & $\begin{array}{c}\mathrm{P} \\
-8.89 \mathrm{P} \\
\mathrm{P} \\
-8.31 \mathrm{P} \\
-9.40 \mathrm{P}\end{array}$ & $\begin{array}{c}\mathrm{P} \\
0.55 \mathrm{P} \\
\mathrm{P} \\
0.46 \mathrm{P} \\
-0.44 \mathrm{P}\end{array}$ \\
\hline $\begin{array}{l}5194 \\
5195 \\
5350 \\
5395 \\
5457\end{array}$ & $\begin{array}{l}85 \\
85 \\
\\
84 \\
26\end{array}$ & $\begin{array}{l}\text { Sbc } \\
\text { I0 } \\
\text { SBb } \\
\text { Sb } \\
\text { Scd }\end{array}$ & $\begin{array}{r}28 \pm 4 \\
20^{\mathrm{b}} \\
9 \pm 3 \\
16 \pm 5 \\
24 \pm 6\end{array}$ & $\begin{array}{l}-10.5^{\mathrm{a}} \\
-\overline{12.3^{\mathrm{a}}} \\
-11.9 \\
-10 . \mathrm{a}^{\mathrm{a}}\end{array}$ & $\begin{array}{l}-8.14 \mathrm{P} \\
\mathbf{P} \\
-9.75 \\
-9.14 \mathrm{P} \\
-8.26\end{array}$ & $\begin{array}{l}0.39 \mathrm{P} \\
\mathrm{P} \\
0.30 \\
0.39 \mathrm{P} \\
0.09\end{array}$ \\
\hline $\begin{array}{l}5474 \\
5480 \\
5544 \\
5545 \\
5774\end{array}$ & $\begin{array}{l}199 \\
199\end{array}$ & $\begin{array}{c}\text { Scd } \\
\text { Sc } \\
\text { SBO/a } \\
\text { Scd } \\
\text { Sd }\end{array}$ & $\begin{array}{r}18 \pm 2 \\
41 \pm 4 \\
1 \pm 2 \\
30 \pm 8 \\
33 \pm 4\end{array}$ & $\begin{array}{l}-11.7 \\
-12.0 \\
-\overline{12.4} \\
-11.9\end{array}$ & $\begin{array}{c}-9.94 \\
-9.62 \mathrm{P} \\
-10.33 \mathrm{P} \\
\mathbf{P} \\
-8.85 \mathrm{P}\end{array}$ & $\begin{array}{c}-0.34 \\
0.44 \mathrm{P} \\
0.04 \mathrm{P} \\
\mathbf{P} \\
0.77 \mathrm{P}\end{array}$ \\
\hline $\begin{array}{l}5775 \\
5850 \\
5929 \\
5930 \\
5985\end{array}$ & $\begin{array}{l}90 \\
90\end{array}$ & $\begin{array}{l}\text { SBc } \\
\text { SBb } \\
\text { Sab } \\
\text { Sb } \\
\text { Sb }\end{array}$ & $\begin{array}{r}31 \pm 4 \\
3 \pm 2 \\
24 \pm 6 \\
25 \pm 9 \\
0 \pm 3\end{array}$ & $\begin{array}{r}-11.6 \\
-12.7: \\
-12.3: \\
-12.3: \\
-\end{array}$ & $\begin{array}{c}\mathbf{P} \\
-9.96 \\
-9.33 P \\
\mathbf{P} \\
-9.97\end{array}$ & $\begin{array}{c}\mathbf{P} \\
-0.24 \\
0.62 \mathrm{P} \\
\mathbf{P} \\
-0.26\end{array}$ \\
\hline \multicolumn{7}{|c|}{ b) Additional members of Arp sample } \\
\hline $\begin{array}{l}2535 \\
2536 \\
5394^{\mathrm{c}} \\
7753 \\
7752 \\
5953 \\
5954 \\
5421 \mathrm{~A} \\
5421 \mathrm{~B} \\
275 \\
2445 \\
2719 \mathrm{~A} \\
2719 \mathrm{~B} \\
6052^{\mathrm{d}} \\
5278\end{array}$ & $\begin{array}{r}82 \mathrm{~A} \\
82 \mathrm{~B} \\
84 \mathrm{~B} \\
86 \mathrm{~A} \\
86 \mathrm{~B} \\
91 \mathrm{~A} \\
91 \mathrm{~B} \\
111 \mathrm{~A} \\
111 \mathrm{~B} \\
140 \mathrm{~B} \\
143 \\
202 \mathrm{~A} \\
202 \mathrm{~B} \\
209 \\
239 \mathrm{~A}\end{array}$ & $\begin{array}{c}\text { Sc } \\
\text { SBc } \\
\text { SBb } \\
\text { Sbc } \\
\text { I0 } \\
\text { Sa } \\
\text { Scd } \\
\text { S } \\
\text { S } \\
\text { Scd } \\
\text { Sc } \\
\text { Im } \\
\text { Im } \\
\text { Sc } \\
\text { Sb }\end{array}$ & $\begin{array}{c}41 \pm 7 \\
55 \pm 8 \\
32 \pm 4 \\
11 \pm 4 \\
70 \pm 10 \\
53 \pm 5 \\
62 \pm 6 \\
10 \pm 3 \\
4 \pm 3 \\
81 \pm 10 \\
45 \pm 7 \\
110 \pm 30 \\
235 \pm 60 \\
150 \pm 20 \\
50 \pm 6\end{array}$ & $\begin{array}{l}-11.5 \\
=12.1: \\
=12.4 \\
-12.6 \\
=12.4: \\
=11.8 \\
-11.9 \\
=12.8: \\
-13.5: \\
=11.8 \\
-11.7 \\
-12.5 \\
=12.2 \\
=11.7 \\
-12.4\end{array}$ & $\begin{array}{c}-9.81 \mathrm{P} \\
\mathbf{P} \\
-9.14 \mathrm{P} \\
-9.48 \mathrm{P} \\
\mathbf{P} \\
-9.24 \mathrm{P} \\
\mathbf{P} \\
-10.27 \mathrm{P} \\
\mathbf{P} \\
-9.57 \mathrm{P} \\
-9.73 \mathrm{P} \\
-10.04 \mathrm{P} \\
\mathbf{P} \\
-9.41 \mathrm{P} \\
-9.99 \mathrm{P}\end{array}$ & $\begin{array}{l}0.30 \mathrm{P} \\
\mathrm{P} \\
0.39 \mathrm{P} \\
0.68 \mathrm{P} \\
\mathrm{P} \\
1.03 \mathrm{P} \\
\mathbf{P} \\
0.40 \mathrm{P} \\
\mathbf{P} \\
0.45 \mathrm{P} \\
0.54 \mathrm{P} \\
0.42 \mathrm{P} \\
\mathbf{P} \\
0.83 \mathrm{P} \\
0.32 \mathrm{P}\end{array}$ \\
\hline
\end{tabular}


TABLE II. (continued)

\begin{tabular}{|c|c|c|c|c|c|c|}
\hline NGC/IC & Arp & Type & $\begin{array}{c}W_{\lambda}(\mathrm{H} \alpha+\mathrm{N} \text { II }) \\
(\AA)\end{array}$ & $\begin{array}{c}\log f(\mathrm{H} \alpha+\mathrm{N} \text { II }) \\
\left(\mathrm{erg} \mathrm{cm}^{-2} \mathrm{~s}^{-1}\right)\end{array}$ & $\begin{array}{c}\log f(\mathrm{FIR}) \\
\left(\mathrm{erg} \mathrm{cm}^{-2} \mathrm{~s}^{-1}\right)\end{array}$ & $\log \frac{L(\text { FIR })}{L(B)}$ \\
\hline $\begin{array}{l}5279 \\
5257 \\
5258 \\
4038 / 9^{d} \\
5427 \\
5426 \\
2881 \mathrm{~A} \\
2881 \mathrm{~B} \\
2799^{\mathrm{c}} \\
7714 \\
5754 \\
5755 \\
7469 \\
\text { IC 5283 } \\
1241 \\
1242\end{array}$ & $\begin{array}{c}239 \mathrm{~B} \\
240 \mathrm{~A} \\
240 \mathrm{~B} \\
244 \\
271 \mathrm{~A} \\
271 \mathrm{~B} \\
275 \mathrm{~A} \\
275 \mathrm{~B} \\
283 \mathrm{~B} \\
284 \mathrm{~A} \\
297 \mathrm{~A} \\
297 \mathrm{~B} \\
297 \mathrm{C} \\
298 \mathrm{~A} \\
298 \mathrm{~B} \\
304 \mathrm{~A} \\
304 \mathrm{~B}\end{array}$ & $\begin{array}{c}\mathrm{Sa} \\
\mathrm{Sb} \\
\mathrm{Sb} \\
\mathrm{SBm} \\
\mathrm{Sc} \\
\mathrm{Sc} \\
\mathrm{Sc} / \mathrm{Im} \\
\mathrm{Sc} \\
\mathrm{SBm} \\
\mathrm{SBb} \\
\mathrm{SBb} \\
\mathrm{SB} \\
\mathrm{I} ? \\
\mathrm{Sa} \\
\mathrm{Scd} \\
\mathrm{SBb} \\
\mathrm{SBc}\end{array}$ & $\begin{array}{l}36 \pm 5 \\
80 \pm 10 \\
50 \pm 6 \\
95 \pm 10 \\
20 \pm 4 \\
16 \pm 4 \\
48 \pm 6 \\
30 \pm 4 \\
56 \pm 6 \\
150 \pm 15 \\
36 \pm 4 \\
18 \pm 4 \\
43 \pm 5 \\
97 \pm 10 \\
14 \pm 4 \\
26 \pm 5 \\
28 \pm 5\end{array}$ & $\begin{array}{l}-13.0 \\
-11.9 \\
-12.4 \\
-10.8^{\mathrm{a}} \\
-11.8 \\
-12.1: \\
-12.5 \\
-12.5 \\
-12.6 \\
-11.5 \\
-12.6 \\
-12.9: \\
-13.0 \\
-11.5: \\
-13.0 \\
-12.1: \\
-12.7:\end{array}$ & $\begin{array}{c}\mathbf{P} \\
-9.19 \mathrm{P} \\
\mathbf{P} \\
-8.60 \mathrm{P} \\
-9.30 \mathrm{P} \\
\mathbf{P} \\
-10.12 \mathrm{P} \\
\mathbf{P} \\
-8.96 \mathrm{P} \\
-9.28 \mathrm{P} \\
-9.74 \mathrm{P} \\
\mathbf{P} \\
\mathbf{P} \\
-8.89 \mathrm{P} \\
\mathbf{P} \\
-9.62 \mathrm{P} \\
\mathbf{P}\end{array}$ & $\begin{array}{c}\mathbf{P} \\
1.18 \mathbf{P} \\
\mathbf{P} \\
0.89 \mathrm{P} \\
0.36 \mathrm{P} \\
\mathbf{P} \\
0.50 \mathrm{P} \\
\mathbf{P} \\
1.18 \mathrm{P} \\
0.97 \mathbf{P} \\
0.93 \mathrm{P} \\
\mathbf{P} \\
\mathbf{P} \\
1.15 \mathrm{P} \\
\mathbf{P} \\
0.42 \mathrm{P} \\
\mathbf{P}\end{array}$ \\
\hline
\end{tabular}

${ }^{\mathrm{a}} \mathrm{H} \alpha$ flux does not include entire galaxy.

${ }^{\mathrm{b}} \mathrm{H} \alpha$ data uncertain due to foreground contamination from M51.

${ }^{c}$ Faint companion to galaxy in complete sample.

${ }^{d}$ Both galaxies (or merger) measured together.

total FIR flux, using the convention described in the IRAS Explanatory Supplement (1985). The fluxes are listed for each detected galaxy (or pair) in the interacting galaxy samples in Table II. We have also listed the FIR fluxes normalized to the total flux in the Johnson $B$ passband, using blue magnitudes from de Vaucouleurs et al. (1976) or from $\mathrm{Pa}$ per I, and the $B$ band photometric calibration from Johnson (1969).

A serious limitation of the IRAS data for this application is its relatively poor angular resolution at 60 and $100 \mu \mathrm{m}$. Only 20 members of the complete-pairs sample are clearly resolved, and virtually none of the other, strongly perturbed pairs in the Arp sample are resolved. We obtained coadded scan data for several of the close pairs, but again the resolution proved to be inadequate in most cases. In Table II we have listed the fluxes of individual galaxies if they are in pairs which are resolved by IRAS, or the total flux of the pairs if the individual components were not resolved. The latter are denoted by the suffix 'P' in Table II. In contrast, individual FIR fluxes could be obtained for virtually all of the galaxies in the control samples, a difference that seriously complicates any comparison of the FIR properties of interacting and noninteracting galaxies.

\section{RESULTS}

\section{a) Ho Equivalent Widths}

Figure 2 shows the distribution of $\mathrm{H} \alpha$ equivalent widths (EWs) for the two samples of close pairs and for the KK control sample, integrated over all Hubble types. The $\mathrm{H} \alpha$ $\mathrm{EW}$, defined as the ratio of the total emission-line flux of the galaxy to the underlying continuum intensity, essentially measures the current massive SFR, normalized to the red luminosity of the galaxy, and as such is a useful distanceindependent and luminosity-independent index of relative star-formation activity. For the sake of orientation, the median EW for Sb galaxies is roughly $10 \AA$, while for Sc galaxies it is approximately $30 \AA$. The reader is referred to Kennicutt (1983) and Kennicutt and Kent (1983) for general discussions of the $\mathrm{H} \alpha$ emission properties of normal galaxies.

Figure 2 demonstrates that both samples of interacting galaxies, especially the Arp sample, exhibit stronger average $\mathrm{H} \alpha$ emission than isolated galaxies. The differences are especially pronounced in the high-EW tails of the distributions. While galaxies with $\mathrm{EW}>60 \AA$ occur only rarely among single field galaxies, they comprise over a quarter of the interacting galaxies we observed. These results, especially for the Arp sample, are in excellent agreement with a study of $\mathrm{H} \alpha$ emission in very strongly interacting and merging systems by Bushouse (1986), and we confirm his conclusion that global SFRs are systematically enhanced in the strongly interacting systems.

Figure 3 compares the samples on a type-by-type basis, and here the dispersion in emission among the interacting galaxies is even more pronounced. Again the Arp sample is

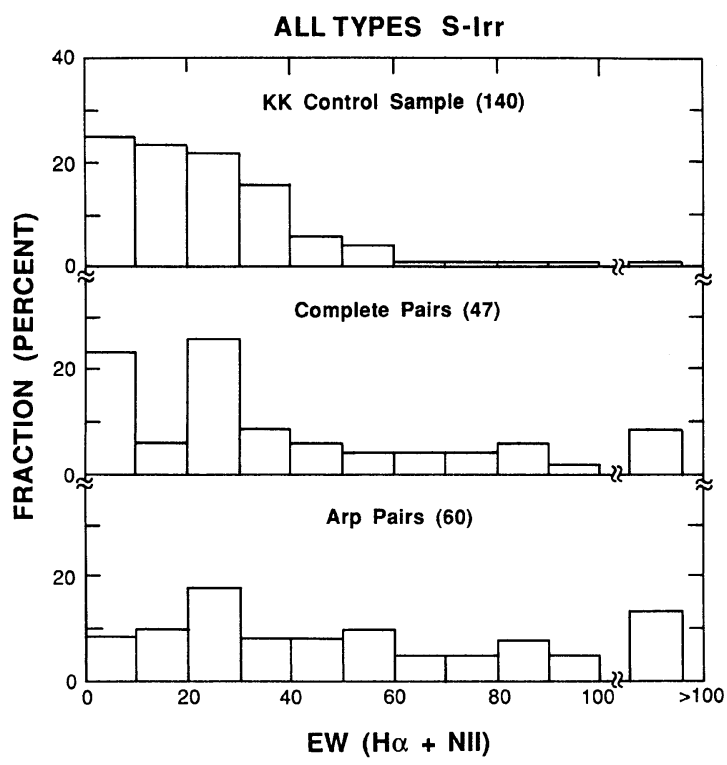

FIG. 2. Distribution of integrated $\mathrm{H} \alpha+[\mathrm{N}$ II $]$ emission-line equivalent widths for single and interacting galaxies. Numbers in parentheses denote the number of galaxies in each sample. 

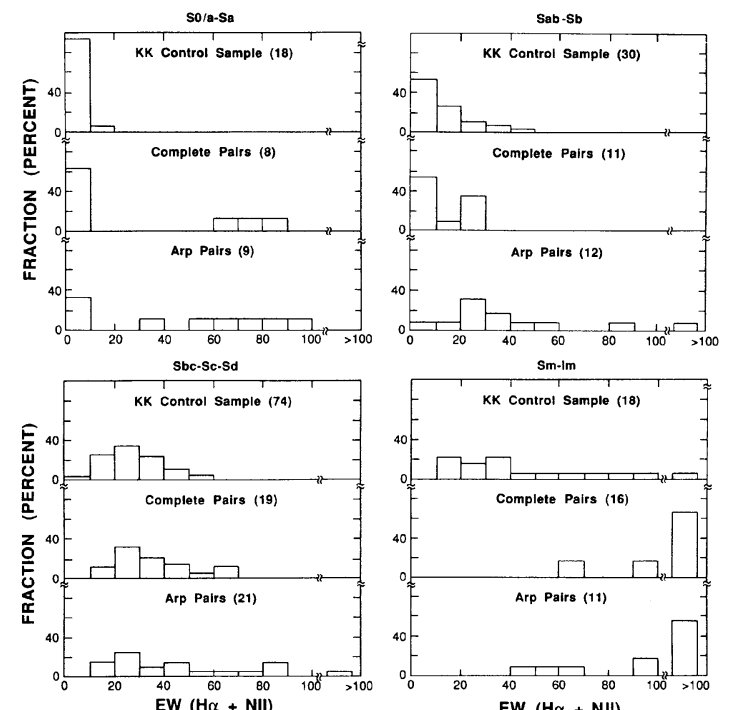

FIG. 3. Distribution of integrated $\mathrm{H} \alpha+[\mathrm{N}$ II] emission-line equivalent widths for single and interacting galaxies, for individual Hubble types.

extreme in its properties; the strong dependence of $\mathrm{H} \alpha$ emission on Hubble type which characterizes normal field galaxies is virtually absent in that sample. As will be discussed in Sec. III $b$, the total emission in most of these galaxies is dominated by disk star formation. The only exceptions are several of the interacting SO/a-Sa galaxies in Fig. 3, which possess bright Seyfert or starburst nuclei. Hence most of the differences in $\mathrm{H} \alpha \mathrm{EW}$ which are evident in Figs. 2 and 3 arise from differences in the disk star-formation rates.

The large range of $\mathrm{H} \alpha$ emission properties among the galaxies we observed implies that the effects of interactions on the global star-formation activity are highly variable. Although many of the interacting galaxies show evidence of star-formation bursts, a large fraction exhibit essentially normal SFRs. This is illustrated by Table III, which lists the median EWs of the different samples. The median EW of the complete-pairs sample is only slightly higher than that of the control sample, indicating that a majority of those interacting galaxies are currently experiencing little or no change in their SFRs. Starbursting galaxies, although present in significant numbers in both samples of interacting systems, comprise only a small fraction of the complete sample.

There are several possible physical causes for this large variation in induced star-formation activity. For example, the SFR is probably sensitive to the orbital properties of the interaction; wide pairs and pairs with retrograde orbits are not likely to disturb each other's disks, while pairs undergo-

TABLE III. Median equivalent widths.

\begin{tabular}{lccc}
\hline \hline & \multicolumn{3}{c}{ EW (H $\alpha+\mathrm{N}$ II $) \AA$} \\
\cline { 2 - 4 } & Control sample & Complete pairs & Arp pairs \\
\hline All types & 21 & 27 & 46 \\
S0/a;Sa & 2 & 4 & 53 \\
Sab-Sb & 9 & 9 & 38 \\
Sbc-Sd & 26 & 33 & 38 \\
Sm-Im & 35 & 140 & 124 \\
\hline \hline
\end{tabular}

ing close, slow, and/or prograde passages are likely to suffer strong disk perturbations. If orbital properties are the dominant determinant of the SFR, then the slight differences between the $\mathrm{H} \alpha$ properties of the complete pairs and control samples would suggest that the range in orbital parameters is quite large. The induced SFR is probably also sensitive to the ambient properties of the disks themselves, especially in relatively distant, weak encounters. Yet another source of variability may be the time scale for the induced star formation; if it is short compared to the lifetime of the gravitational encounter, then a complete sample of pairs will exhibit a large range of observed star-formation properties.

In order to test whether the interaction kinematics can dominate the SFR in these galaxies, we have investigated the correlation between the EWs of individual members of the same pair, and the dependence of the EW on pair separation. Figure 4 shows the $\mathrm{H} \alpha$ emission of each member of a pair plotted against the corresponding emission of its companion. Two parameters are plotted, the absolute EW and an ' $\mathrm{H} \alpha$ excess' $r$, defined as the EW of a galaxy divided by the median EW for isolated galaxies of the same Hubble type (Table III). If the SFR were dominated by the orbital properties of the interaction, we would expect to observe a correlation between the emission levels within pairs, while if the induced SFR were dominated by the ambient properties of individual galaxies, we would expect little or no correlation. The pairs observed here, especially those with unusually strong starformation activity ( $\mathrm{EW} \geqslant 50 \AA$ ), do show a significant degree of correlation in their emission properties. A similar correlation has been observed between the colors of close pairs, the "Holmberg effect" (Holmberg 1958; Madore 1986). Part of this effect is probably due to the tendency of binary galaxies to possess similar morphological types (Holmberg 1937; Page 1975), but the correlation observed here in $\mathrm{H} \alpha$, especially at high EWs, must largely reflect interaction-induced star formation.

Figure 5 illustrates the dependence of emission on projected pair separation (in units of the harmonic mean galaxy radius). We restrict this comparison to the complete sample, in order to avoid any bias in the Arp sample to close, active star-forming systems. Again, we observe a very large scatter, which is partly due to projection effects, and is partly real. Galaxies with very strong emission ( $E W>50 \AA)$ are usually members of close pairs, while galaxies with weaker $\mathrm{H} \alpha$ emission occur at all separations. Application of a KolmogorovSmirnoff test to the EW distributions of two subsamples with separations closer than and greater than 2 radii shows a systematic difference at the $90 \%$ confidence level. Similar results have been observed for galaxy colors by Madore (1986). These results indicate that many strong starbursts are triggered by close galaxy-galaxy encounters, but that such interactions by themselves are neither necessary nor sufficient conditions for such star-formation bursts.

At the same time, the SFRs in the strongly interacting galaxies appear to be largely decoupled from the internal parameters which regulate the SFR in normal field galaxies. It has already been shown in Fig. 3, for example, that the $\mathrm{H} \alpha$ EWs of the galaxies in the Arp sample are only weakly dependent on morphological type. Another illustration of this effect is shown in Fig. 6, which shows the dependence of the $\mathrm{H} \alpha \mathrm{EW}$ on $\mathrm{H}$ i content. The parameter plotted is the ratio of the $\mathbf{H}$ I mass of each galaxy, taken from Davis and Seaquist (1983) for the interacting galaxies and from Fisher and Tully (1981), Bottinelli et al. (1982), and Huchtmeier (1982) 

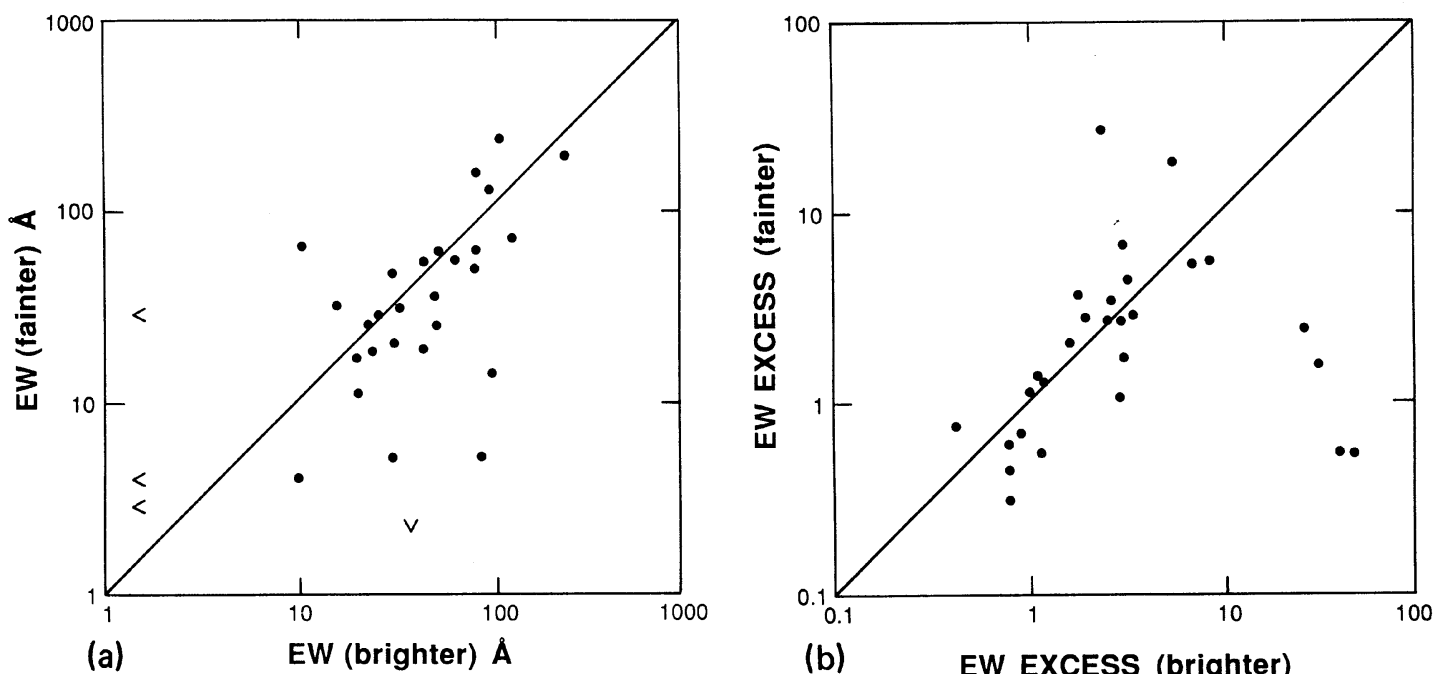

FIG. 4. Dependence of integrated $\mathrm{H} \alpha+[\mathrm{N} \mathrm{II}]$ emission of an interacting galaxy on the corresponding emission of its companion. In each case the brightest member (in the blue) is plotted along the abscissa. (a) Emission-line equivalent widths. (b) Equivalent-width excess factor, as defined in the text.

for the KK control sample, normalized to the square of the optical diameter, as listed by de Vaucouleurs et al. (1976). We chose to use this pseudo surface density as an index of $\mathrm{H} \mathrm{I}$ content, rather than the more conventional $\mathscr{M}(H) / L(B)$ ratio, because the latter is sensitive to luminosity evolution in the starbursting systems. Figure 6 demonstrates that $\mathrm{H} \alpha$ emission is loosely correlated with $\mathrm{H}$ I content among the single galaxies, but this correlation is virtually absent in the interacting systems. Most of the interacting galaxies have much higher $\mathrm{H} \alpha$ EWs than field galaxies with similar $\mathrm{H}_{\mathrm{I}}$ contents, and have SFRs that are essentially independent of their total gas contents (at least their $\mathrm{H}$ I content).

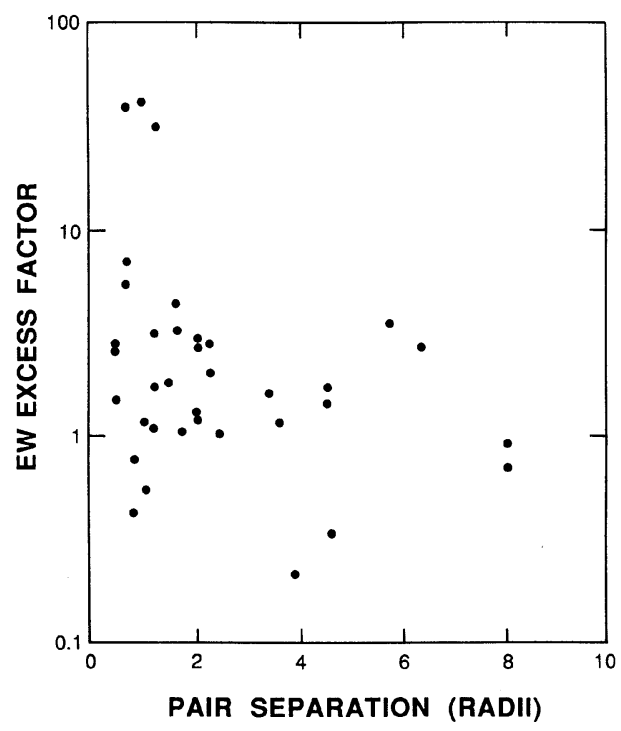

FIG. 5. Dependence of $\mathrm{H} \alpha+[\mathrm{N} \mathrm{II}]$ emission of an interacting galaxy on the projected distance to its nearest companion. Separation is in units of the harmonic mean galactic radius. See the text for a definition of the equivalentwidth excess factor.
To summarize, the interacting galaxies we studied exhibit a large range of responses to interactions, but we can clearly identify two asymptotic physical regimes. At one extreme are galaxies with relatively normal SFRs. The $\mathrm{H} \alpha$ emission in these galaxies is essentially uncorrelated with any identifiable interaction parameter. It is likely that most of the observed $\mathrm{H} \alpha$ emission represents the underlying, steady-state star-formation activity, and this SFR is dictated largely by the internal properties of the disk, independent of the (usually weak) interactions. A majority of the galaxies in a com-

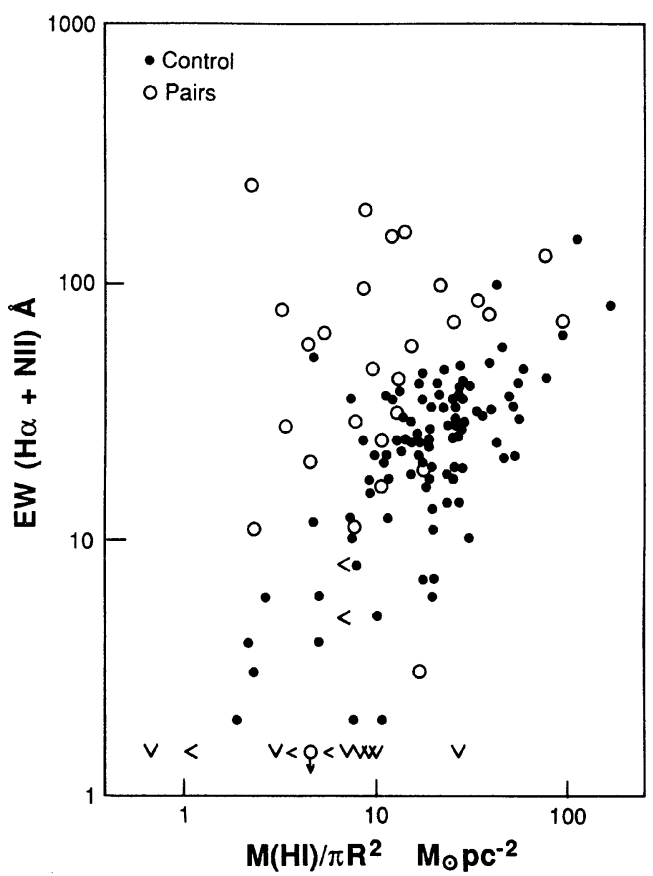

FIG. 6. Relationship between integrated $\mathrm{H} \alpha+[\mathrm{N}$ II] emission-line equivalent width on neutral-hydrogen content for single and interacting galaxies. 
plete sample of close pairs will fall into this regime. At the other extreme are galaxies with abnormally strong $\mathrm{H} \alpha$ emission. The EWs of these galaxies are only weakly correlated with the properties of their parent galaxies, such as Hubble type or $\mathbf{H}$ I content, and much more strongly correlated with the properties of the interactions themselves, as might be expected if the observed emission were dominated by interaction-induced star formation. Although such galaxies are rare in a volume-limited sample, many of the best-known interacting systems fall into this regime.

\section{b) Ho Luminosities and Nuclear Emission}

The $\mathrm{H} \alpha$ luminosities provide additional information about the star formation in the interacting galaxies. Figure 7 shows a comparison of $\mathrm{H} \alpha$ luminosities for the interacting (dark symbols) and single (light symbols) Sc-Irr galaxies. Enhanced emission in the interacting galaxies occurs over the entire range of absolute magnitude, except possibly at faint magnitudes where our sample is too small. Several interacting galaxies possess $\mathrm{H} \alpha$ luminosities that are higher than any of the galaxies in the KK survey, but the brightest is only three times more luminous than normal Sc I galaxies such as M101 or NGC 1232. Hence we do not find any optical analogs to the superluminous interacting/merging galaxies which are observed in the far infrared (Houck et al. 1985). This is not surprising, given the low space density of the FIR sources, the short estimated lifetimes of the bursts, and the association of such sources with mergers (e.g., Joseph and Wright 1985), rather than with distinct pairs of galaxies.

Figure 8 shows a comparison of the integrated $\mathrm{H} \alpha+[\mathrm{N}$ II] fluxes of the interacting galaxies with their nuclear fluxes. The latter were taken from the nuclear spectrophotometry in Paper I, or measured directly from the $\mathrm{H} \alpha$ images; in both cases an aperture of $4.7^{\prime \prime}$ was used. In most of the interacting galaxies the nuclear contribution to the integrated emission is small; the median fraction is $13 \%$, and in all but a few galaxies it is less than $25 \%$. Hence the integrated emission is usually dominated by disk star formation. The fraction of $\mathrm{H} \alpha$ emission arising from the nuclear regions is very similar to that $(\sim 10 \%)$ observed in the radio continuum (Hummel 1981).

Although the fractional contribution of nuclear emission to the total $\mathrm{H} \alpha$ luminosities of the interacting galaxies is small, it is approximately three times higher than the average of $4 \%$ in isolated spirals (Kennicutt and Kent 1983). This suggests that the nucleus (or its immediate environment) is more sensitive than the disk to the effects of the interactions. This effect was first observed at radio wavelengths by Hummel (1980, 1981), who found that the main effect of interactions was to enhance the radio emission of the central source, not the disk. Bushouse (1986) has studied the distribution of $\mathrm{H} \alpha$ emission in a sample of strongly interacting systems, and also observes an unusually strong concentration of the $\mathrm{H} \alpha$ emission to the central regions. As discussed in Paper I and Bushouse (1986), the 'nuclear' emission regions are often spatially extended, and this implies that enhanced emission is occurring in both the unresolved nucleus itself and in the immediately surrounding disk. A more detailed study of this question will be published elsewhere (Keel et al. 1987).

The same data can be used to investigate whether the levels of nuclear and disk activity are correlated. Figure 9 shows the relationship between nuclear and disk EWs in the program galaxies, including galaxies in the control sample with available nuclear spectrophotometry (Keel 1983). Here we have plotted the net disk EWs, in order to eliminate any bias of the total EW from strong nuclear emission. Among galaxies with relatively normal emission ( $E W \leqslant 30-40 \AA$ ), including most of the control sample, there is little or no correlation between the nuclear and disk EWs. The two types of emission do appear to be correlated in the most active galaxies, however. Almost all of the galaxies with strong disk starbursts $(E W>400 \AA$ ) also exhibit unusually strong nuclear emission. (The only exceptions are a few late-type spirals and irregulars which do not posssess identifiable optical nuclei, and hence are not plotted in Figs. 8 and 9.) Apparently, any perturbation that is strong enough to trigger a burst of star formation in the disk will also transfer enough gas to the central regions of the galaxy to enhance the nuclear emission as well.

While strong nuclear emission almost always accompanies strong disk emission in the interacting galaxies we studied, the converse does not hold; some of the most active nuclei in our sample are embedded in disks that are virtually devoid of massive star formation. There are at least two possible explanations for this effect. Most of these latter galaxies are early-type, gas-poor spirals, and perhaps it is easier to trigger star formation and/or nonthermal activity in the nu-

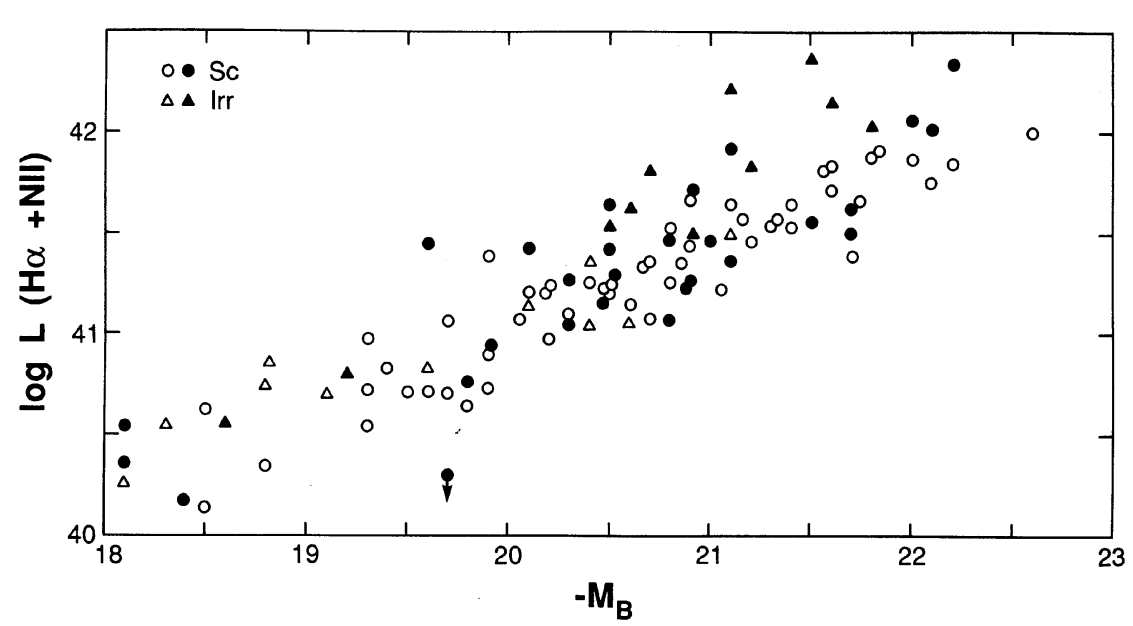

FIG. 7. Relationship between total $\mathrm{H} \alpha$ $+\left[\mathrm{N}_{\text {II }}\right]$ luminosity and absolute blue luminosity for late-type single (light symbols) and interacting (dark symbols) galaxies. 


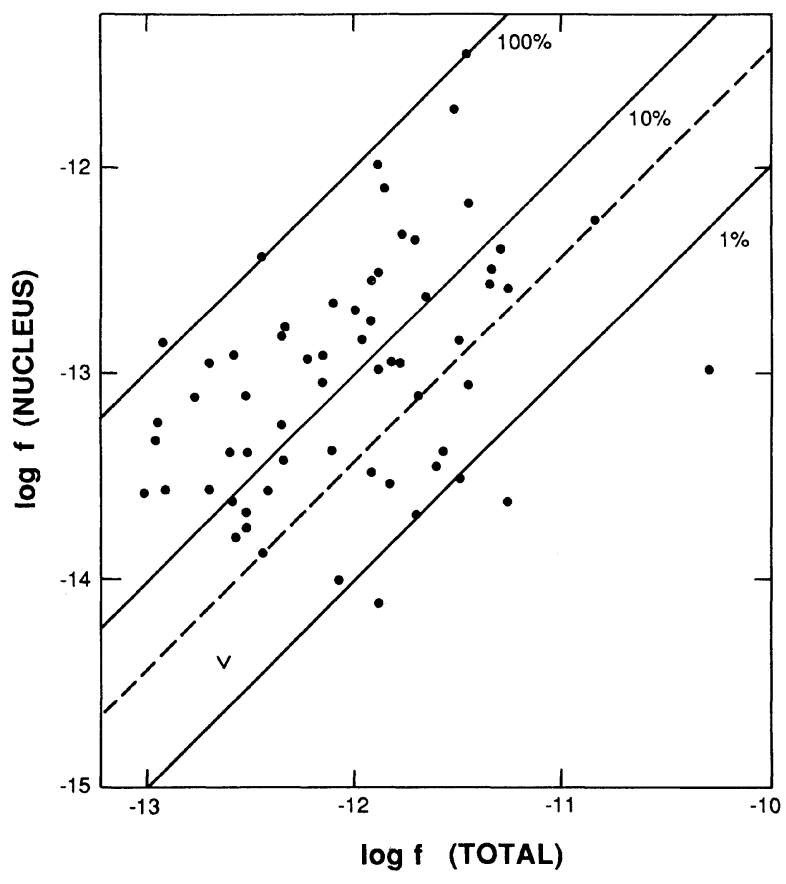

FIG. 8. Relationship between total $\mathrm{H} \alpha+\left[\mathrm{N}_{\text {II }}\right]$ emission-line flux and nuclear emission-line flux for the interacting galaxies. The three solid lines indicate fractional nuclear contributions of $1 \%, 10 \%$, and $100 \%$ of the total flux. The dashed line indicates the median fractional nuclear contribution for single galaxies in the control sample.

clear regions than in the disks of such galaxies. Another possibility is that the effect arises from widely differing time scales for the nuclear and disk activities. Models by Loose $e t$ al. (1982) and Scalo and Struck-Marcell (1986) indicate that star formation in a given galactic component will respond to a disturbance on a time scale that is related to the

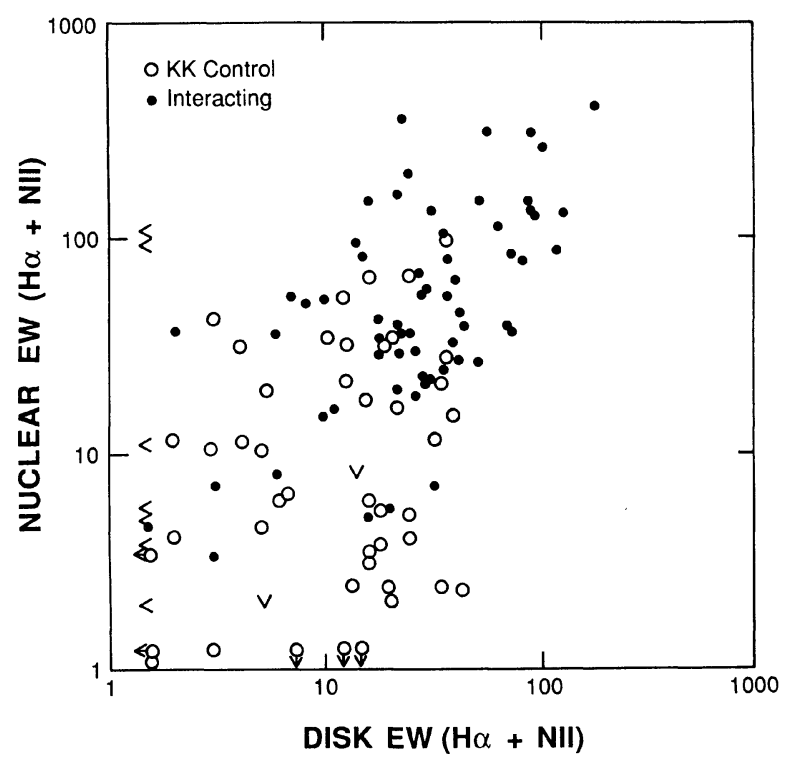

FIG. 9. Relationship between nuclear and disk $\mathrm{H} \alpha+[\mathrm{N}$ II $]$ emissionline equivalent widths for single (dark symbols) and interacting (light symbols) galaxies. dynamical time scale in that component. Such time scales are relatively short in the nuclear regions, and perhaps the nuclei respond more quickly than the disks to a tidal disturbance.

\section{c) Infrared Emission}

Infrared emission in galaxies is often associated with star formation (e.g., Rieke and Lebofsky 1979; de Jong et al. 1984), and thus if the excess $\mathrm{H} \alpha$ emission observed in interacting systems is due to star formation, we would expect a corresponding enhancement in the FIR luminosities of these galaxies. Evidence for enhanced IR emission in interacting galaxies has been reported by Lonsdale et al. (1984), Joseph et al. (1984), Cutri and McAlary (1985), and Bushouse (1986). Here we use the IRAS FIR data to test whether this is a general property of interacting galaxies.

As mentioned earlier, any analysis of the IRAS fluxes of individual members of close pairs is severely handicapped by the limited spatial resolution of that survey at 60 and 100 $\mu \mathrm{m}$. About half of the pairs in our complete sample and most of the Arp sample are not resolved.

Another serious problem is the uncertain relationship between the flux of a galaxy in the IRAS passbands and its star-formation rate. Detailed spectral decompositions of the FIR emission in galaxies by several investigators (e.g., Cox et al. 1986; Walterbos and Schwering 1987; Persson and Helou 1987) indicate that only part of the FIR emission is associated with massive star formation, and this fraction may vary considerably among different types of galaxies. In view of these ambiguities, we have chosen to limit our analysis to a statistical comparison of the FIR properties of the interacting and noninteracting galaxies, as a consistency check on our interpretations of the optical data.

Since much of the IRAS data for the interacting galaxies consist of integrated pair fluxes, we first compare the distributions of total FIR emission from pairs rather than from individual galaxies. Fluxes for the interacting pairs were taken directly from the IRAS data. The total (pair) FIR flux was then combined with the total blue luminosity to derive an average $L($ FIR $) / L(B)$ ratio, following the procedures described in Sec. II. For the comparison sample, we generated an artificial set of pairs by matching each member of the $\mathrm{KK}$ control sample (now including E/SO galaxies) with another randomly selected galaxy. In order to match the interacting-pair samples as much as possible, we required that the difference in recessional velocity of the artificially paired galaxies be less than $600 \mathrm{~km} \mathrm{~s}^{-1}$, and required that each artificial pair contain at least one spiral or irregular galaxy. Each member of the KK sample was paired with two other galaxies in this way, in order to generate a smoother distribution of pair fluxes.

The results of this comparison are shown in Fig. 10. The reader is referred to de Jong et al. (1984) and Hunter et al. (1986) for general discussions of the behavior of the $L(\mathrm{FIR}) / L(B)$ ratio in normal galaxies. Many of the same features observed in the comparison of $\mathrm{H} \alpha \mathrm{EWs}$ (Fig. 2) are seen in the FIR distributions as well. The median $L$ (FIR)/ $L(B)$ ratio is systematically higher in the interacting galaxies $(1.45 \times$ for the complete sample and $2.2 \times$ for the Arp sample), and there is a substantial population of infraredbright interacting pairs which is virtually absent from the control sample. The average enhancements we observe in the FIR are similar to those observed in $\mathrm{H} \alpha$. We do not observe an excess of FIR emission relative to $\mathrm{H} \alpha$ in interacting sys- 


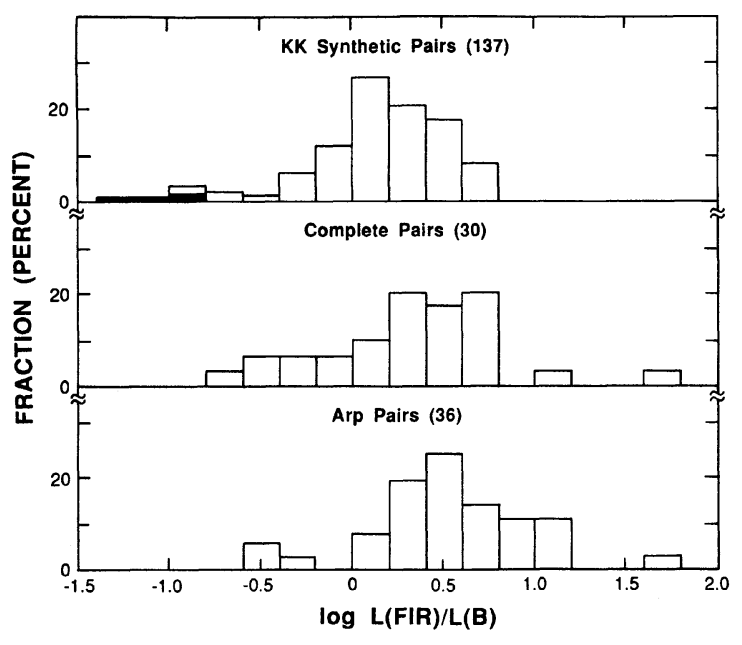

FIG. 10. Distribution of total far-infrared luminosity, normalized to total blue luminosity, of interacting pairs and an artificial set of pairs generated randomly from the single-galaxy control sample. Shaded areas denote upper limits.

tems, as has been reported by Bushouse (1986), but this may be due to a much larger fraction of strongly interacting systems in Bushouse's survey.

Figure 11 shows a crude comparison of the FIR properties of individual galaxies. For galaxies in the two control samples and for roughly half of the complete-pairs sample, the FIR fluxes were obtained directly from the IRAS data. For each unresolved pair, we apportioned the total FIR flux among its components by setting their relative FIR fluxes equal to their relative $\mathrm{H} \alpha$ fluxes. The $\mathrm{H} \alpha$ and FIR luminosities of individual field galaxies are roughly correlated (Dennefeld et al. 1986), so this arbitrary procedure is probably satisfactory for comparing the general propertiesof the FIR flux distributions. Values for the complete sample which were estimated in this way are indicated in Fig. 11. The Arp

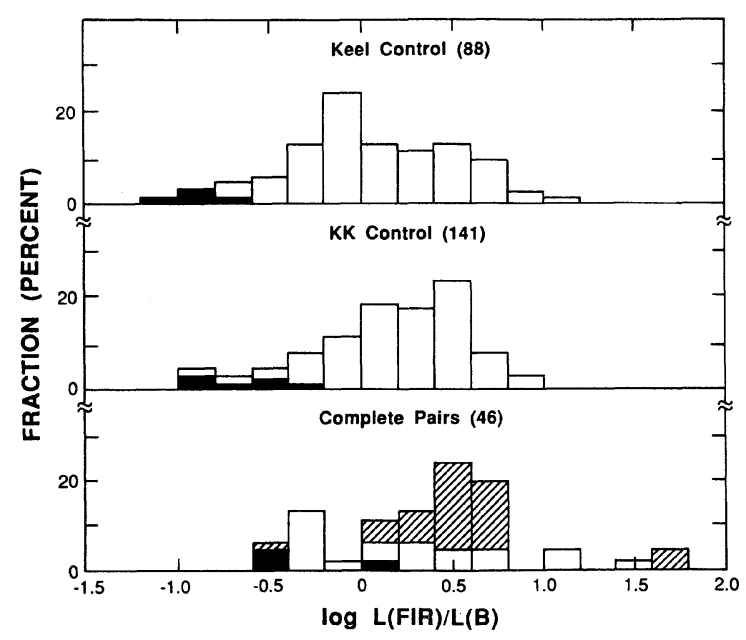

FIG. 11. Distribution of total far-infrared luminosity, normalized to total blue luminosity, for individual isolated and interacting galaxies. Shaded areas denote upper limits. Hatched areas denote members of unresolved pairs where the relative $\mathrm{H} \alpha$ fluxes were used to estimate the relative infrared fluxes within the pair. sample is not included in this comparison, because nearly all its members are unresolved.

Both control samples are shown in Fig. 11, and the similarity in their FIR properties is reassuring; if anything, the $\mathrm{KK}$ control sample which was used for the $\mathrm{H} \alpha$ analysis is slightly biased toward more active star-forming galaxies. The interacting galaxies show most of the same qualitative features which are evident in the comparisons in Figs. 2 and 10 ; enhanced emission on average and an excess of very active systems. Any further interpretation would require higher-resolution data.

\section{STAR-FORMATION RATES AND EVOLUTIONARY PROPERTIES}

Larson and Tinsley (1978) interpreted the $U B V$ colors of interacting galaxies in terms of a simple star-formation-burst model. The $\mathrm{H} \alpha$ data enable us to test the consistency of this picture, and to place some rudimentary constraints on the properties of the star-formation bursts.

Figure 12(a) shows the relationship between the $\mathrm{H} \alpha \mathrm{EWs}$ and corrected $B-V$ colors (de Vaucouleurs et al. 1976) for the single and the interacting galaxies. This diagram is very useful for isolating galaxies with star-formation bursts because the $\mathrm{H} \alpha$ emission, produced by stars with lifetimes of a few million years, is sensitive to the very recent SFR, while the broadband colors, dominated by the ratio of intermediate-mass main-sequence stars to giants, evolve more slowly to changes in the SFR. H $\alpha$ emission is strongly correlated with color in normal S-Irr galaxies, and most of the interacting galaxies follow this same general relation. A considerable number of the interacting galaxies, however, possess unusually high $\mathrm{H} \alpha$ EWs for their color, as would be expected for galaxies experiencing strong transient star-formation bursts.

In order to interpret these observations, we have computed a set of $\mathrm{H} \alpha$ and color-evolution models, following the methods described in Kennicutt (1983), and a summary of these models is shown in Fig. 12(b). The shaded line shows the $\mathrm{H} \alpha$-color relation for a set of standard disk models with solar metallicity, an "extended Miller-Scalo" IMF (Kennicutt 1983), and a variable star-formation history (e.g., Searle et al. 1973; Larson and Tinsley 1978). The shading indicates the approximate effect of extinction variations. This model, from Kennicutt (1983), provides an excellent fit to the observed $U B V$ colors and $\mathrm{H} \alpha \mathrm{EWs}$ of normal disk galaxies. Also shown in Fig. 12(b) are lines representing the colors of three galaxies with star-formation bursts superimposed. These were modeled by adding to each galaxy a $10^{7}$ year-old population with continuous star formation. Each line represents the effect of varying the relative strength of this star-formation burst; the symbols denote enhancements of 2,10 , and 100 times the prior SFR in the disk. The properties of galaxies with longer star-formation bursts will lie between the limits defined by the $10^{7} \mathrm{yr}$ burst models and the underlying (steady state) disk models.

Comparison of Figs. 12(a) and 12(b) shows that the simple burst models can satisfactorily account for the properties of most of the interacting galaxies in our sample, confirming the interpretation of the galaxy colors by Larson and Tinsley (1978). (The only exceptions are NGC 3227, a red Seyfert galaxy, and M82, which is heavily reddened.) For most of the interacting galaxies the inferred bursts are relatively modest, with enhancements in the SFR of 2-3 times the prior rate in the disk, and durations of order $10^{7}-10^{8} \mathrm{yr}$. The most active star-forming galaxies in our sample require 

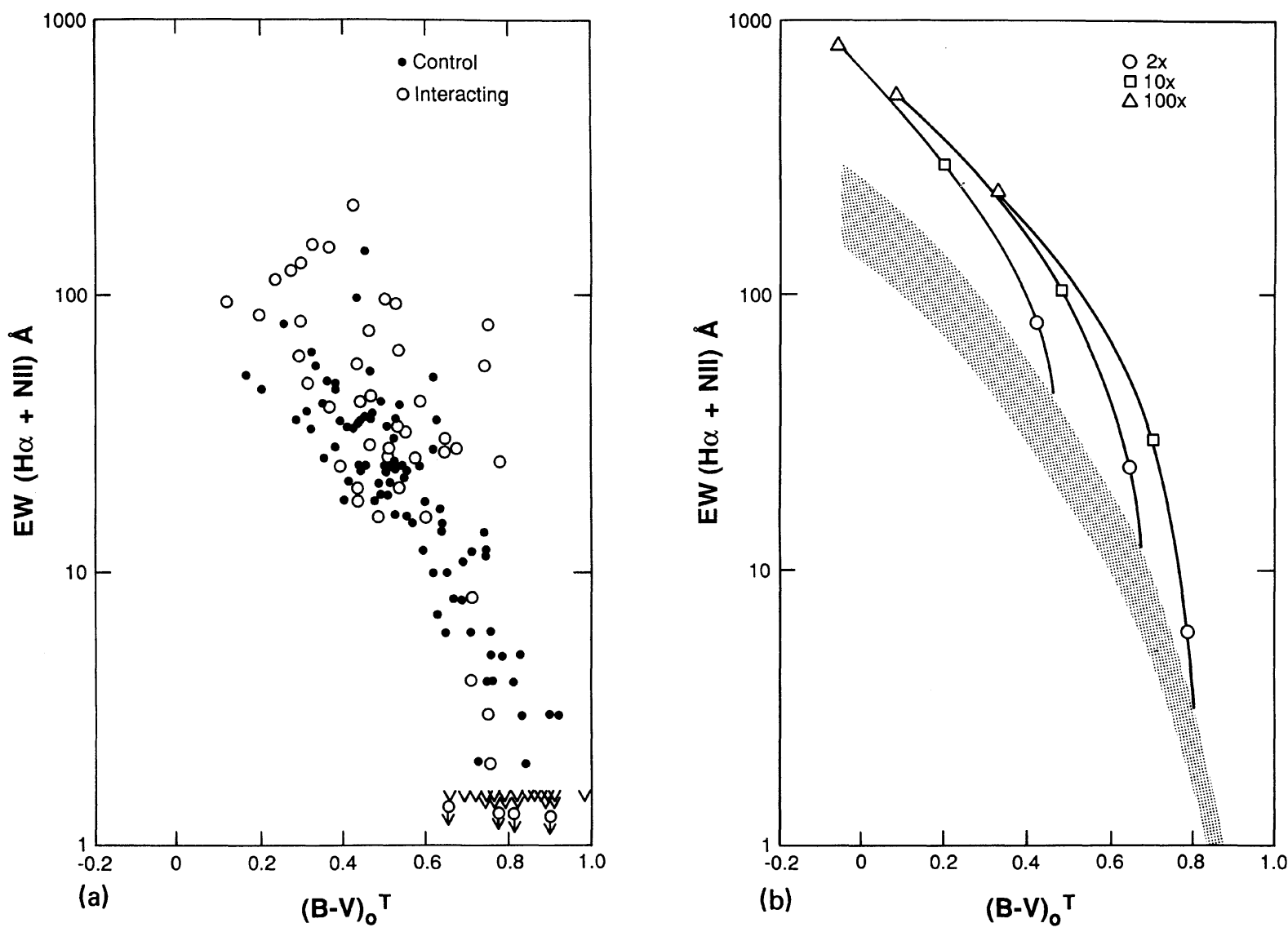

FIG. 12. (a) Relationship between integrated $\mathrm{H} \alpha+[\mathrm{N} \mathrm{II}]$ equivalent width and integrated color for single (dark symbols) and interacting (light symbols) galaxies. (b) Galaxy evolution models. The shaded region is a steady-state disk evolution model, while the lines show the effects of a superimposed star-formation burst. Symbols denote the strength of the burst, relative to the prior underlying star-formation rate in the disk.

bursts of 5-30 times the prior SFR (assuming that they were normal Sb-Sc-Irr galaxies prior to the interaction), with durations of order $10^{7} \mathrm{yr}$. These results are in good agreement with the earlier $U B V$ models of Larson and Tinsley (1978).

The H $\alpha$ fluxes in Table II can be combined with simple photoionization models such as those in Kennicutt (1983) or Gallagher et al. (1984), in order to obtain rough estimates of the SFRs in the interacting galaxies. Applying the calibration of Kennicutt (1983) to the data (including a mean correction for extinction) yields SFRs of $0.1-10 \mathscr{M}_{\odot} \mathrm{yr}^{-1}$ in massive $\left(\geqslant 10 \mathscr{M}_{\odot}\right)$ stars, and an estimated range in total SFRs of $\sim 1-60 \mathscr{M}_{\odot} \mathrm{yr}^{-1}$. Although the SFRs in the brightest galaxies are prodigious, they are only a factor 3-5 higher than is typical in luminous normal Sc galaxies.

An important ingredient in these calculations is the presumed shape of the IMF. The results above have been derived under the assumption that the IMF in the star-formation bursts is identical to that in the underlying disks (with a roughly Salpeter slope above $\left.1 \mathscr{M}_{\odot}\right)$. There is evidence from the work of Huchra (1977), Gehrz et al. (1983), and Auguarde and Lequeux (1985), however, which suggests that the IMF in starbursting galaxies is systematically enriched in massive stars. Although the normal IMF burst models in Fig. 12(b) can reproduce the observed $\mathrm{H} \alpha$ EWs and colors of most of the interacting galaxies, the high EWs in the most active systems can only be contrived with short bursts $\left(\sim 10^{7} \mathrm{yr}\right)$ and very little extinction. Both of the latter are unrealistic, and consequently the strong $\mathrm{H} \alpha$ emission is most likely produced by an IMF which is selectively enriched in OB stars. Hence we tentatively conclude that the massivestar-enriched IMFs previously observed in a few unusually luminous starbursting systems are probably a common property of strong interaction-induced star-formation bursts. Unfortunately, it is not possible with the colors and $\mathrm{H} \alpha$ EWs alone to fit a model with both variable IMF and variable burst properties. If the slope of IMF in the burst is shallow, the resulting SFRs will be lower, and the inferred durations of the bursts will be longer than discussed above.

The star-formation rates can be combined with the burst properties derived earlier, in order to estimate the total stellar mass produced in the starbursts. The most luminous and active galaxies in our sample, with SFRs of a few times 10 $\mathscr{M}_{\odot} \mathrm{yr}^{-1}$ and burst lifetimes of $2-3 \times 10^{7} \mathrm{yr}$, will produce a total of $10^{9}-10^{10} \mathscr{M}_{\odot}$ of stars, corresponding to a few percent or less of the total disk mass. In most of the galaxies we studied, the mass of stars formed will be lower; less than $1 \%$ of the disk mass. Hence we conclude that while the interactions may cause major transient perturbations in the evolution of the galaxies involved, the star formation in most bursts is minor in comparison to the past star formation in the disks of these galaxies. The stellar mass involved in these 
bursts is also small when compared to the total supplies of $\mathrm{H}$ I available for star formation. In the most extreme case, NGC 3690 + IC 694, the available supplies of H I (Davis and Seaquist 1983) and molecular gas (Sanders et al. 1986) will fuel the present SFR for at least $2 \times 10^{8} \mathrm{yr}$; in the other galaxies for which $\mathrm{H}$ I data are available, the gas supplies would sustain even the present SFRs for more than $10^{9} \mathrm{yr}$.

It has been suggested that externally induced star formation may produce a significant fraction of all of the massive stars in S-Irr galaxies (Condon 1983), and we can use our results to test this hypothesis. If we operationally define 'interacting' galaxies to be those which meet the criteria established for our complete-pairs sample, then approximately $10 \%$ of all S-Irr galaxies in a magnitude-limited sample are members of such interacting systems. The galaxies in our complete-pairs sample have an average $\mathrm{H} \alpha$ luminosity that is approximately 1.6 times brighter than the members of the control sample, and if we ascribe all of the difference to interactions, we conclude that the interactions are responsible for only $6 \pm 3 \%$ of the total present massive-star formation in luminous S-Irr galaxies. The considerable uncertainties in this calculation arise mostly from the arbitrary definition of interactions, and from uncertainty in the matching of the complete and KK control samples, but it is clear in any case that externally induced star formation is a minor contribution, at least at the present epoch.

Finally, we should briefly mention some aspects of these results which are not easily understood. As mentioned earlier, the time scales we infer for the star-formation bursts (order $10^{7} \mathrm{yr}$ ) are uncomfortably short when compared to the expected durations of the tidal encounters themselves. A related problem is the absence of any blue but $\mathrm{H} \alpha$-weak galaxies in our sample. Since the $U B V$ colors of a galaxy decay more slowly after a burst than the $\mathrm{H} \alpha$ emission, we expect to observe several galaxies abnormally low in $\mathrm{H} \alpha$ emission for their colors. These problems would be relieved if the bursts have a shallow IMF, as discussed earlier. Another possibility is that the star-formation bursts decay gradually over a period of order $10^{8} \mathrm{yr}$, following a rapid initial rise. If the latter effect is present, we are probably observing a considerable fraction of the interacting systems during the relatively quiescent stages following a much more active star-formation burst.

\section{CONCLUSIONS}

Both the integrated $\mathrm{H} \alpha$ emission and the FIR emission are systematically enhanced in the interacting galaxies studied here, and these results provide strong confirmation that tidal interactions can enhance the star-formation rate in galactic disks. The degree of enhancement varies enormously, however, indicating that the induced star formation is sensitive to both the detailed kinematic properties of the interactions and to the ambient properties of the galactic disks.

We have found that the inferred star-formation properties of a sample of interacting galaxies are very sensitive to the observational selection criteria used. In particular, compilations of peculiar galaxies such as the Arp (1966) atlas appear to be very heavily biased toward the most active starforming systems; virtually every starbursting system in our complete sample is also a member of the Arp catalog. Part of this selection appears to be physical, insofar as the peculiar galaxies generally include the most strongly perturbed systems, but much of it appears to reflect a strong bias to unusu- ally high-surface-brightness, active systems. Hence, while the well-known peculiar systems may provide a useful indication of the extremes in observed star-formation properties, they do not provide a representative sample of interacting systems, especially for the study of star formation.

Our results explain what had been a puzzling inconsistency between the studies of Larson and Tinsley (1978), Joseph et al. (1984), and Lonsdale et al. (1984), who had found evidence for strong star-formation bursts in interacting galaxies, and the results of Hummel $(1980,1981)$, who found no evidence for abnormal disk star formation. The former studies were concentrated on peculiar systems, mostly from the Arp atlas, and the strong effects which were attributed to interactions are partly real and partly a manifestation of the selection effect discussed above. Hummel, on the other hand, studied a sample of nearby pairs very similar to the complete sample in this paper; the subtle differences which we observed in our complete sample would probably have escaped detection in his radio survey.

Our $\mathrm{H} \alpha$-derived star-formation rates are in excellent agreement with the widely quoted burst models of Larson and Tinsley (1978). Star-formation bursts of modest strength (involving a few percent or less of the disk mass in newly formed stars) and duration (order $10^{7} \mathrm{yr}$ ) can reproduce the properties of all but the most extreme systems in our sample. The latter galaxies, with unusually strong $\mathrm{H} \alpha$ emission, are best understood if the initial mass function in the burst is enriched in OB stars.

Our results suggest that interactions are not a major influence on the evolution of most galaxies at the present epoch. The relatively small number of active systems ( $\leqslant 10 \%$ $15 \%$ ) in our complete sample indicates that major interaction-induced perturbations in the SFR are either very rare or very brief. In environments where interactions or mergers are common, however, such as in the cores of rich clusters or at early cosmological epochs, such interactions could be much more important.

Finally, our results confirm the importance of interacting galaxies for understanding the most luminous star-forming systems. The most active interacting galaxies in our sample possess $\mathrm{H} \alpha$ and FIR luminosities that rarely occur (if every) in isolated galaxies, a distinction that is shared by the starforming nuclei in interacting systems (e.g., Paper I; Balzano 1983; Bushouse 1986). Although the most active systems we studied represent only a small minority of a volume-limited sample of close pairs of galaxies, they may dominate the statistics of surveys that are selected on the basis of strong ultraviolet, infrared, radio, or optical line emission.

We wish to thank Jeff Pedelty and George Will for help with the KPNO observations, and Jeanette Barnes for assistance with the data reduction. R. C. K. acknowledge the support of NSF grant AST81-11711A01 and an Alfred P. Sloan Foundation Fellowship. J. M. H. and R. C. K. acknowledge the support of NATO International Travel Grant 0592/82. This research was funded in part under the IRAS extended mission program by JPL contract 957243 . The Mount Lemmon telescope is supported in part by NSF grant AST84-20347. Finally, the Kapteyn telescope is operated on the island of La Palma by the Royal Greenwich Observatory, on behalf of the SERC. 


\section{REFERENCES}

Arp, H. (1966). Atlas of Peculiar Galaxies (California Institute of Technology, Pasadena).

Auguarde, R., and Lequeux, J. (1985). Astron. Astrophys. 147, 273.

Balzano, V. A. (1983). Astrophys. J. 268, 602.

Beichman, C. A., Neugebauer, G., Habing, H. J., Clegg, P. E., and Chester, T. J., editors (1985), IRAS Catalogs and Atlases, Explanatory Supplement (U. S. GPO, Washington, DC).

Bottinelli, L., Gouguenheim, L., and Paturel, G. (1982). Astron. Astrophys. Suppl. 47, 171.

Bushouse, H. A. (1986). Ph.D. thesis, University of Illinois.

Carlson, J. B. (1977). Ph.D. thesis, University of Maryland.

Cohen, J. G. (1976). Astrophys. J. 203, 587.

Condon, J. J. (1983). Astrophys. J. Suppl. 53, 459.

Condon, J. J., Condon, M. A., Gisler, G., and Puschell, J. J. (1982). Astrophys. J. 252, 102.

Cox, P. N., Kugel, E., and Mezger, P. G. (1986). Astron. Astrophys. 155, 380.

Cutri, R. M., and McAlary, C. W. (1985). Astrophys. J. 296, 90.

Dahari, O. (1984). Astron. J. 89, 966.

Dahari, O. (1985). Astrophys. J. Suppl. 57, 643.

Davis, L. E., and Seaquist, E. R. (1983). Astrophys. J. Suppl. 53, 269.

de Jong, T., et al. (1984). Astrophys. J. Lett. 279, L67.

Dennefeld, M., Karoji, H., and Belfort, P. (1986). In Star Forming Dwarf Galaxies and Related Objects, edited by D. Kunth, T. X. Thuan, and J. Tran Thanh Van (Editions Frontieres, Gif sur Yvette, France), p. 351.

de Vaucouleurs, G., de Vaucouleurs, A., and Corwin, H. G. (1976). Second Reference Catalog of Bright Galaxies (University of Texas, Austin).

Fisher, J. R., and Tully, R. B. (1981). Astrophys. J. Suppl. 47, 139.

Gallagher, J. S., Hunter, D. A., and Tutukov, A. V. (1984). Astrophys. J. 284, 544.

Gehrz, R. D., Sramek, R. A., and Weedman, D. W. (1983). Astrophys. J. 267, 551.

Heidman, J., and Kalloghlian, A. T. (1973). Astrofizika 9, 71.

Holmberg, E. (1937). Lunds. Ann. No. 6.

Holmberg, E. (1958). Medd. Lund. Astron. Obs. Ser. 2, No. 136

Houck, J. R., et al. (1985). Astrophys. J. Lett. 290, L5.

Huchra, J. P. (1977). Astrophys. J. 217, 928.

Huchtmeier, W. K. (1982). Astron. Astrophys. 110, 121.

Hummel, E. (1980). Astron. Astrophys. 89, L1.

Hummel, E. (1981). Astron. Astrophys. 96, 111.
Hunter, D. A., Gillett, F. C., Gallagher, J. S., Rice, W. L., and Low, F. J. (1986). Astrophys. J. 303, 171.

IRAS Point Source Catalog (1985). Joint IRAS Science Working Group (U. S. GPO, Washington, DC).

Johnson, H. L. (1966). Annu. Rev. Astron. Astrophys. 4, 193.

Joseph, R. D., Meikle, W. P. S.; Robertson, N. A., and Wright, G. S. (1984). Mon. Not. R. Astron. Soc. 209, 111.

Joseph, R. D., and Wright, G. S. (1985). Mon. Not. R. Astron. Soc. 214, 87. Keel, W. C. (1983). Astrophys. J. Suppl. 52, 229.

Keel, W. C., Kennicutt, R. C., Hummel, E., and van der Hulst, J. M. (1985). Astron. J. 90, 708 (Paper I).

Kennicutt, R. C. (1983). Astrophys. J. 272, 54.

Kennicutt, R. C., Bothun, G. D., and Schommer, R. A. (1984). Astron. J. 89, 1279.

Kennicutt, R. C., and Keel, W. C. (1984). Astrophys. J. Lett. 279, L5.

Kennicutt, R. C., and Kent, S. M. (1983). Astron. J. 88, 1094.

Larson, R. B., and Tinsley, B. M. (1978). Astrophys. J. 219, 46

Lonsdale, C. J., Persson, S. E., and Matthews, K. (1984). Astrophys. J. 287, 95.

Loose, H. H., Kugel, E., and Tutukov, A. V. (1982). Astron. Astrophys. 105,342 .

Madore, B. F. (1986). In Spectral Evolution of Galaxies, edited by C. Chiosi and A. Renzini (Reidel, Dordrecht), p. 97.

Nilson, P. (1973). Uppsala General Catalog of Galaxies, Uppsala Astron. Obs. Ann. 6.

Page, T. (1975). In Galaxies and the Universe, edited by A. Sandage, M. Sandage, and J. Kristian (University of Chicago, Chicago), p. 541.

Persson, C. J., and Helou, G. (1987). Astrophys. J. (in press).

Sanders, D. B., et al. (1986). Astrophys. J. Lett. 305, L5.

Scalo, J. M., and Struck-Marcell, C. (1986). Astrophys. J. 301, 77.

Searle, L., Sargent, W. L. W., and Bagnuolo, W. G. (1973). Astrophys. J. $179,427$.

Soifer, B. T., et al. (1984). Astrophys. J. Lett. 278, L71.

Stocke, J. T. (1978). Astron. J. 83, 348

Sulentic, J. W. (1976). Astrophys. J. Suppl. 32, 171.

van Albada, T. S. (1986). Preprint.

Walterbos, R. A. M., and Schwering, P. B. W. (1987). Astron. Astrophys. (in press).

Wasilewski, A. J. (1983). Astrophys. J. 272, 68. 
PLATE 63
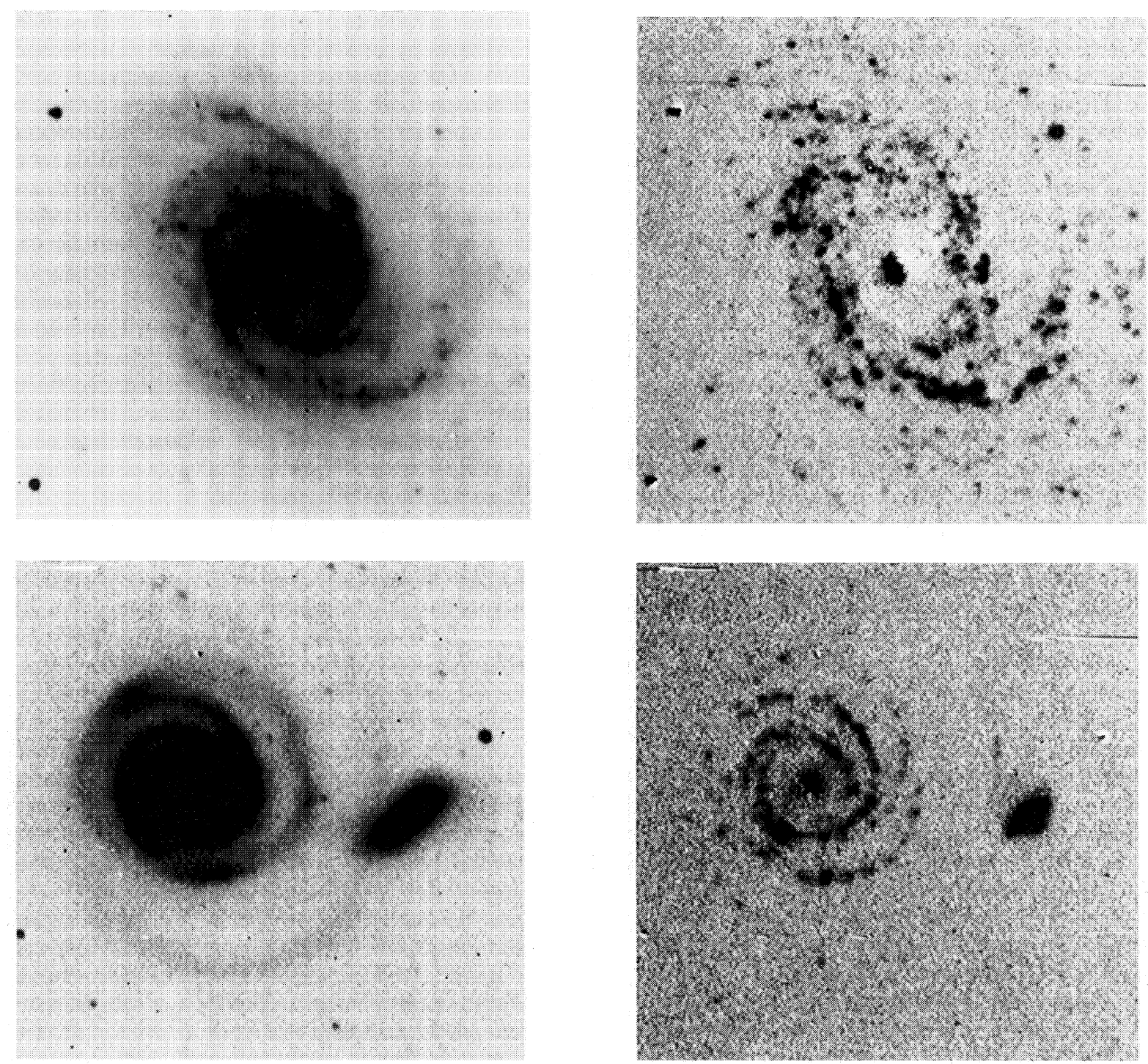

FIG. 1. Continuum (left) and $\mathrm{H} \alpha+[\mathrm{N} \mathrm{II}]$ (right) emission-line images of interacting galaxies. Top: NGC 5350, a member of the complete-pairs sample. Bottom: NGC 5754 and Arp 297C. Another companion, NGC 5755, is out of the field.

Kennicutt et al. (see page 1012) 